PROCEEDINGS OF THE

AMERICAN MATHEMATICAL SOCIETY

Volume 134, Number 4, Pages 1237-1248

S 0002-9939(05)08062-7

Article electronically published on August 29, 2005

\title{
MAPPING SPACES AND HOMOLOGY ISOMORPHISMS
}

\author{
NICHOLAS J. KUHN, \\ WITH AN APPENDIX BY GREG ARONE AND NICHOLAS J. KUHN \\ (Communicated by Paul Goerss)
}

\begin{abstract}
Let $\operatorname{Map}(K, X)$ denote the space of pointed continuous maps from a finite cell complex $K$ to a space $X$. Let $E_{*}$ be a generalized homology theory. We use Goodwillie calculus methods to prove that under suitable conditions on $K$ and $X, \operatorname{Map}(K, X)$ will send an $E_{*}$-isomorphism in either variable to a map that is monic in $E_{*}$ homology. Interesting examples arise by letting $E_{*}$ be $K$-theory, the finite complex $K$ be a sphere, and the map in the $X$ variable be an exotic unstable Adams map between Moore spaces.
\end{abstract}

\section{Introduction AND MAIN RESUlts}

Let $K$ and $X$ be pointed spaces, with $K$ homotopy equivalent to a finite cell complex, and then let $\operatorname{Map}(K, X)$ denote the space of pointed continuous maps from $K$ to $X$. Fixing $K$, this includes many important constructions on $X$. For example, $\operatorname{Map}\left(S^{n}, X\right)=\Omega^{n} X$, the $n^{\text {th }}$ loopspace of $X$, and $\operatorname{Map}\left(S_{+}^{1}, X\right)=\mathcal{L} X$, the free loopspace on $X$.

Suppose $E_{*}$ is a generalized homology theory. A fundamental problem is to try to determine to what extent $E_{*}(\operatorname{Map}(K, X))$ might be determined by $E_{*}(X)$. This is difficult, and has a long history, even when $E_{*}$ is ordinary homology with field coefficients and $K=S^{1}$.

We consider a related problem. A map $f: X \rightarrow Y$ will be called an $E_{*^{-}}$ isomorphism if $E_{*}(f)$ is an isomorphism. One can ask to what extent does $\operatorname{Map}(K$, preserve $E_{*}$-isomorphisms?

This question is of interest when $E_{*}$ is a nonconnective theory, as the following simple example illustrates: the constant map $c: K(\mathbb{Z} / p, 2) \rightarrow *$ is an isomorphism in complex $K$-theory $K_{*}$, but $\Omega c: K(\mathbb{Z} / p, 1) \rightarrow *$ is not. A much more subtle family of examples has been constructed recently by L. Langsetmo and D. Stanley LS; see Example 1.4 below.

In this paper we use Goodwillie calculus methods to prove the curious result that under suitable conditions on $K$ and $X, \operatorname{Map}(K, X)$ will send an $E_{*}$-isomorphism in either variable to a map that is monic in $E_{*}$ homology.

1.1. The main theorem. We need to define some numerical invariants of spaces. Let $d(K)$ be the minimal $d$ such that $K$ is homotopy equivalent to a $d$-dimensional complex.

Received by the editors September 2, 2004 and, in revised form, November 8, 2004.

2000 Mathematics Subject Classification. Primary 55P35; Secondary 55N20, 55P42.

This research was partially supported by a grant from the National Science Foundation.

(c)2005 American Mathematical Society 
Let $e(K)$ be the minimal $n$ such that there exists a parallelizable $n$-dimensional manifold $M$ (not necessarily compact, and possibly with boundary), together with a closed subcomplex $A$ such that $K$ is homotopy equivalent to $M / A$. For example, $e\left(S^{n}\right)=n$, as $S^{n}=D^{n} / S^{n-1}$.

Let $c(X)$ be the connectivity of $X$.

Let $s(X)$ be the maximal $n$ such that $X$ is homotopy equivalent to an $n$-fold suspension.

Armed with these definitions, we can state our main theorem.

Theorem 1.1. Suppose $e(K) \leq s(X)$ and $d(K) \leq c(X)$.

(1) If $f: X \rightarrow Y$ is an $E_{*}$-isomorphism, then

$$
\operatorname{Map}(K, f): \operatorname{Map}(K, X) \rightarrow \operatorname{Map}(K, Y)
$$

is $E_{*}-$ monic.

(2) If $g: L \rightarrow K$ is an $E_{*}$-isomorphism of finite complexes, then

$$
\operatorname{Map}(g, X): \operatorname{Map}(K, X) \rightarrow \operatorname{Map}(L, X)
$$

is $E_{*}-$ monic.

Corollary 1.2. If $Z$ is connected, and $f: \Sigma^{n} Z \rightarrow Y$ is an $E_{*}$-isomorphism, then $\Omega^{n} f: \Omega^{n} \sum^{n} Z \rightarrow \Omega^{n} Y$ is $E_{*}-$ monic.

Remark 1.3. This corollary seems to be new even when $n=1$. To the best of the author's knowledge, the only results of this sort in the literature are the author's papers [K1, K2] which contain the $n=\infty$ version of the corollary.

Note that $d(K) \leq e(K)$. Furthermore $s(X) \leq c(X)+1$ is always true, and very often $s(X) \leq c(X)$. For example, $s\left(M^{n}(d)\right)=c\left(M^{n}(d)\right)=n-2$, where $M^{n}(d)$ is the Moore space $D^{n} \cup_{d} S^{n-1}$. Thus when the first inequality in the hypotheses of the theorem holds, so usually does the second. In general, $e(K)$ seems hard to compute exactly. The appendix includes some observations of Greg Arone and the author which yield some further explicit calculations, and some general bounds. For example, $e\left(M^{n}(d)\right)=n+1$, and, $e(K) \leq 2 d(k)-1$ for all $K$ with $d(K) \geq 1$.

The numeric hypotheses of our theorem are easy to explain. The condition $d(K) \leq c(X)$ guarantees the strong convergence of the Goodwillie tower of the functor sending a space $X$ to the suspension spectrum $\Sigma^{\infty} \operatorname{Map}(K, X)$. The condition $e(K) \leq s(X)$ implies that there is a filtered configuration space approximation to $\operatorname{Map}(K, X)$, as in work of Bödigheimer [Bö], following McDuff [McD] and May Ma.

When both numeric conditions hold, statement (1) of Theorem 1.1 is proved by using properties of Goodwillie towers to play the two corresponding geometric conditions against each other. Using the existence of Bousfield localization of spaces, statement (2) is then a formal consequence of (1).

The proof of Theorem 1.1 is given in sections 2 and 3 . In section 2, we outline how general calculus theory leads to theorems like ours, while in the shorter section 3 , we specialize to the case in hand.

\subsection{Examples and applications.}

Example 1.4. Let $p$ be an odd prime. For each $(m, n)$ in an explicit infinite list of pairs, with $m \geq 4$ and both $m$ and $(n-m)$ taking on arbitrarily large values, 
Langsetmo and Stanley [LS] construct a $K_{*}$-isomorphism

$$
f: M^{n}(p) \rightarrow M^{m}(p)
$$

such that $\Omega f$ is not a $K_{*}$-isomorphism. For example, with $p=3$, for all $t \geq 1$, one has such a nondurable $K_{*}$-isomorphism $f: M^{4 t}(3) \rightarrow M^{4}(3)$.

It is not hard to deduce that then, for all $j \geq 1$, the 3 -connected cover of $\Omega^{j} f$,

$$
\left(\Omega^{j} f\right)\langle 3\rangle: \Omega^{j} M^{n}(p)\langle 3\rangle \rightarrow \Omega^{j} M^{m}(p)\langle 3\rangle,
$$

is also not a $K_{*}$-isomorphism 1

In contrast, Corollary 1.2 implies that for all $1 \leq j \leq n-2$,

$$
\Omega^{j} f: \Omega^{j} M^{n}(p) \rightarrow \Omega^{j} M^{m}(p)
$$

is $K_{*}$-monic.

Combining these results, we conclude that for $1 \leq j \leq n-5$,

$$
\left(\Omega^{j} f\right)\langle 3\rangle: \Omega^{j} M^{n}(p) \rightarrow \Omega^{j} M^{m}(p)\langle 3\rangle
$$

is $K_{*}$-monic but not $K_{*}$-epic.

Example 1.5. When the homology theory $E_{*}$ is $K(r)_{*}$, the $r^{t h}$ Morava $K$-theory at a prime $p$, then the corollary has the following computational implication.

Let $f: \Sigma^{n} Z \rightarrow Y$ be a $K(r)_{*}$-isomorphism, with $Z$ connected and $n \geq 1$, and let $F$ be the fiber of $f$. The $K(r)_{*}$ bar spectral sequence associated to the principal fibration

$$
\Omega^{n} \Sigma^{n} Z \stackrel{\Omega^{n} f}{\longrightarrow} \Omega^{n} Y \rightarrow \Omega^{n-1} F
$$

converges to $K(r)_{*}\left(\Omega^{n-1} F\right)$ and has

$$
E_{*, *}^{2}=\operatorname{Tor}_{*, *}^{K(r)_{*}\left(\Omega^{n} \Sigma^{n} Z\right)}\left(K(r)_{*}\left(\Omega^{n} Y\right), K(r)_{*}\right) .
$$

By the corollary,

$$
K(r)_{*}\left(\Omega^{n} \Sigma^{n} Z\right) \stackrel{\left(\Omega^{n} f\right)_{*}}{\longrightarrow} K(r)_{*}\left(\Omega^{n} Y\right)
$$

is monic. The map $\left(\Omega^{n} f\right)_{*}$ is in the category of $\mathcal{K} / p$-Hopf algebras studied by Bousfield in [B2, Appendix]. He shows [B2, Thm.10.8] that objects in this category are flat over subobjects, when viewed as algebras. We conclude that the spectral sequence collapses, giving an isomorphism

$$
K(r)_{*}\left(\Omega^{n-1} F\right) \simeq K(r)_{*}\left(\Omega^{n} Y\right) \otimes_{K(r)_{*}\left(\Omega^{n} \Sigma^{n} Z\right)} K(r)_{*}
$$

of $K(r)_{*}$-coalgebras 2

Example 1.6. Suppose $g: L \rightarrow K$ is a $K(r)_{*}$-isomorphism between finite complexes. Let $C$ be the cofiber of $g$. Applying statement (2) of Theorem 1.1 to $\Sigma g$, and reasoning as in the last example, we deduce that, for all $X$ such that $e(K)<s(X)$ and $d(K)<c(X)$, one gets an isomorphism of $K(r)_{*}-$ coalgebras

$$
K(r)_{*}(\operatorname{Map}(C, X)) \simeq K(r)_{*}(\operatorname{Map}(\Sigma L, X)) \otimes_{K(r)_{*}(\operatorname{Map}(\Sigma K, X))} K(r)_{*} .
$$

\footnotetext{
${ }^{1}$ This follows from a theorem of Bousfield [B3. Theorem 11.10], but is easy to prove directly, using the fact that $\tilde{K}_{*}(K(\mathbb{Z} / p, 2)=0$.

${ }^{2} K(r)_{*}$-Hopf algebras if $n>1$.
} 


\section{Goodwillie CALCUlus AND $E_{*}$-ISOMORPhisms}

Let $\mathcal{T}$ denote the category of based spaces, and let $\mathcal{S}$ be a nice model category of spectra, e.g. the category of $S$-modules of [EKMM]. In this section we find conditions on a functor $F: \mathcal{T} \rightarrow \mathcal{S}$ and a space $X$ ensuring that if $f: X \rightarrow Y$ is an $E_{*}$-isomorphism, then $F(f): F(X) \rightarrow F(Y)$ will be $E_{*}$-monic.

2.1. Review of Goodwillie calculus. In the series of papers G1, G2, G3, Tom Goodwillie has developed his theory of polynomial resolutions of homotopy functors. We need to summarize some aspects of Goodwillie's work as they apply to functors $F: \mathcal{T} \rightarrow \mathcal{S} !^{3}$

As is carefully discussed in G2, G3], a functor is said to be polynomial of degree $r$ if it takes strongly homotopy cocartesion $(r+1)$-cubical diagrams to homotopy cartesian cubical diagrams. In [G3], given a functor $F$ from one topological model category to another, Goodwillie proves the existence of a tower $\left\{P_{r} F\right\}$ under $F$ so that $F \rightarrow P_{r} F$ is the universal arrow to a polynomial functor of degree $r$, up to weak equivalence.

The functors $F$ of interest to us in this paper satisfy an additional property: they will be finitary. Here, following G3, Definition 5.10], $F$ is said to be finitary if it commutes with filtered homotopy colimits up to equivalence.

Examination of the construction of $P_{r} F$ shows that $P_{r}$ satisfies the following useful properties.

Lemma 2.1 (Compare with [G3, Proposition 1.7]).

(1) If $F(X) \rightarrow G(X) \rightarrow H(X)$ is a fibration sequence for all $X$, so is $P_{r} F(X) \rightarrow$ $P_{r} G(X) \rightarrow P_{r} H(X)$.

(2) Given natural transformations $F_{1} \rightarrow F_{2} \rightarrow \ldots$, the natural map

$$
\underset{s}{\operatorname{hocolim}} P_{r} F_{s}(X) \rightarrow P_{r}\left(\underset{s}{\operatorname{hocolim}} F_{s}\right)(X)
$$

is an equivalence for all $r$ and $X$.

(3) If $F$ is finitary, so is $P_{r} F$ for all $r$.

The fact that the suspension of a strongly homotopy cocartesian cube is again strongly homotopy cocartesion implies the next property of Goodwillie towers.

Lemma 2.2 (G3. Remark 1.1]). There is a natural equivalence

$$
P_{r}\left(F \circ \Sigma^{d}\right)(X) \simeq\left(P_{r} F\right)\left(\Sigma^{d} X\right) \text {. }
$$

Let $D_{r} F(X)$ be the homotopy fiber of $P_{r} F(X) \rightarrow P_{r-1} F(X) . D_{r} F$ is homogeneous of degree $r$ : it has degree $r$, and $P_{r-1} D_{r} F(X)$ is weakly contractible. (This follows from Lemma 2.1(1); see [G3, Proposition 1.17].) Goodwillie analyzes $D_{r} F$. We need his description when $F$ is also finitary and takes values in a stable model category like $\mathcal{S}$.

Proposition 2.3 (G3, Theorems 3.5, 6.1]). If $F: \mathcal{T} \rightarrow \mathcal{S}$ is finitary, then, for each $r$, there is a spectrum $t_{r}(F)$ with an action of the $r^{\text {th }}$ symmetric group $\Sigma_{r}$, and a natural weak equivalence

$$
D_{r} F(X) \simeq\left(t_{r}(F) \wedge X^{\wedge r}\right)_{h \Sigma_{r}}
$$

\footnotetext{
${ }^{3}$ As surveyed in [K3], Goodwillie's general theory applies to functors $F: \mathcal{C} \rightarrow \mathcal{D}$, where $\mathcal{C}$ and $\mathcal{D}$ are topological or simplicial model categories. As is apparent from the proofs, many of the results in this section would apply to functors with domain $\mathcal{T}$ replaced by any such $\mathcal{C}$, and range $\mathcal{S}$ replaced by a model category that is also stable.
} 
Corollary 2.4. If $F: \mathcal{T} \rightarrow \mathcal{S}$ is finitary, and $f: X \rightarrow Y$ is an $E_{*}$-isomorphism, then $P_{r} F(f): P_{r} F(X) \rightarrow P_{r} F(Y)$ is also an $E_{*}$-isomorphism.

Proof. Standard spectral sequences show that any construction of the form $\left(C \wedge X^{\wedge r}\right)_{h \Sigma_{r}}$ preserves $E_{*}$-isomorphisms in the $X$ variable. The proposition thus implies that the maps on fibers, $D_{r} F(f): D_{r} F(X) \rightarrow D_{r} F(Y)$, are $E_{*}-$ isomorphisms. The proposition then follows by induction on $r$.

Remark 2.5. The corollary is false without the finitary hypothesis. Examples can easily be constructed using homological localization functors, which are homogeneous and linear, but not, in general, finitary.

2.2. Strongly split towers of spectra. Suppose we are given a tower of spectra under another spectrum:

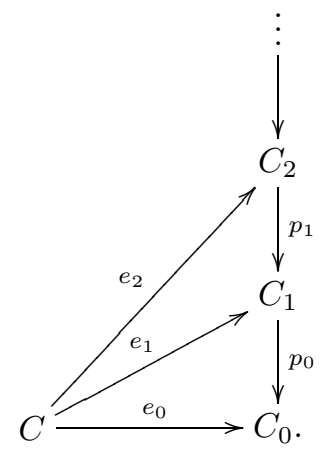

We will say that the tower is strongly convergent if the connectivity of the maps $e_{r}$ goes to infinity as $r$ goes to infinity.

We will say that the tower is strongly split if there exists a homotopy commutative diagram

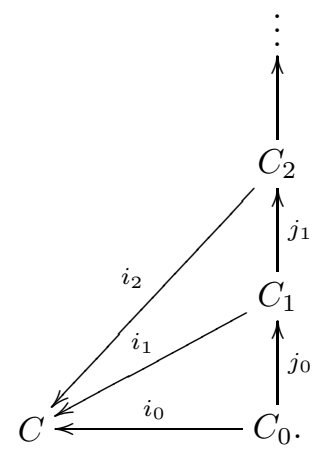

such that, for all $r$, the composite

$$
C_{r} \stackrel{i_{r}}{\longrightarrow} C \stackrel{e_{r}}{\longrightarrow} C_{r}
$$

is an equivalence.

The following lemma is evident.

Lemma 2.6. If a tower as above is both strongly convergent and strongly split, then the induced map

$$
\underset{r}{\operatorname{hocolim}} i_{r}: \underset{r}{\operatorname{hocolim}} C_{r} \rightarrow C
$$


is an equivalence. Thus, if $E_{*}$ is a homology theory, then

$$
\underset{r}{\operatorname{colim}} E_{*}\left(C_{r}\right) \rightarrow E_{*}(C)
$$

is an isomorphism.

Remark 2.7. This lemma says all that we will need to know about strongly split towers for our purposes. However, it is illuminating to note the following. If a tower is strongly split, one can, if needed, modify the splitting data so that $e_{r} \circ i_{r}: C_{r} \rightarrow C_{r}$ is homotopic to the identity. In this case, it will also be true that the composite

$$
C_{r} \stackrel{j_{r}}{\longrightarrow} C_{r+1} \stackrel{p_{r}}{\longrightarrow} C_{r}
$$

will be homotopic to the identity for all $r$. If the tower is also strongly convergent, then there will be a wedge decomposition

$$
C \simeq \bigvee_{r=1}^{\infty} \text { hofiber }\left\{p_{r}: C_{r} \rightarrow C_{r-1}\right\}
$$

\subsection{A useful proposition.}

Proposition 2.8. Let $F: \mathcal{T} \rightarrow \mathcal{S}$ be finitary. Suppose the Goodwillie tower of $F$ is both strongly convergent and strongly split when evaluated at a space $X$. Then, if $f: X \rightarrow Y$ is an $E_{*}$-isomorphism, then $F(f): F(X) \rightarrow F(Y)$ is $E_{*}-$ monic.

Proof. Let $i_{r}: P_{r} F(X) \rightarrow F(X)$ and $j_{r}: P_{r} F(X) \rightarrow P_{r+1} F(X)$ denote the maps splitting the tower $\left\{P_{r} F(X)\right\}$. Suppose $f: X \rightarrow Y$ is an $E_{*}$-isomorphism, and consider the diagram

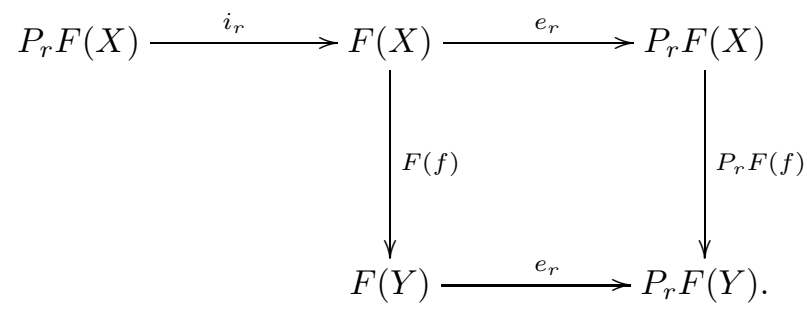

By assumption, the top composite is an equivalence, and thus is an $E_{*}$-isomorphism. Since $f$ is an $E_{*}$-isomorphism, so is the right vertical map, by Corollary 2.4. We conclude that $E_{*}(F(f))$ is monic when restricted to the image of $E_{*}\left(i_{r}\right)$. But $E_{*}(F(X))$ is the colimit over $r$ of these images, by Lemma 2.6, and thus $E_{*}(F(f))$ is also monic.

To apply this proposition, we need criteria ensuring that a Goodwillie tower $\left\{P_{r} F(X)\right\}$ strongly splits. This is the topic of our next two subsections.

2.4. Goodwillie towers of functors with polynomial filtration. Say that a functor $C: \mathcal{T} \rightarrow \mathcal{S}$ has a polynomial filtration if it is filtered by functors $F_{0} C \rightarrow$ $F_{1} C \rightarrow \ldots$ such that

$$
\underset{r}{\text { hocolim }} F_{r} C(X) \rightarrow C(X)
$$

is an equivalence, and the homotopy cofiber functor

$$
F_{r} C / F_{r-1} C
$$

is homogeneous of degree $r$ for all $r$. 
The following lemma is well-known folk knowledge.

Lemma 2.9. In this situation, the composite

$$
F_{r} C(X) \rightarrow C(X) \rightarrow P_{r} C(X)
$$

is an equivalence for all $r$ and $X$. It follows that the Goodwillie tower $\left\{P_{r} C(X)\right\}$ will be strongly split.

Proof. We have a homotopy commutative diagram with rows that are cofibration sequences

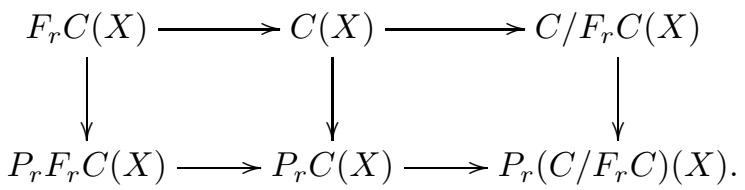

As $F_{r} C$ has degree $r$, the left vertical map is an equivalence. If we check that $P_{r}\left(C / F_{r} C\right)(X) \simeq *$, then the bottom left map will be an equivalence, and we will be done. To check this we have

$$
P_{r}\left(C / F_{r} C\right)(X) \simeq \underset{s}{\operatorname{hocolim}} P_{r}\left(F_{s} C / F_{r} C\right)(X) \simeq *,
$$

as $P_{r}\left(F_{s} C / F_{r} C\right)(X) \simeq *$ for $s \geq r$.

2.5. Stable natural equivalences. Call a natural transformation $\Theta(X): C(X) \rightarrow$ $G(X)$ a stable equivalence if it is an equivalence for all suitably connected spaces $X$.

Goodwillie notes in [G3, Remark 1.1] that his construction of $P_{r} F$ depends only on the behavior of $F$ on highly connected spaces. To say this more precisely, we first recall that $P_{r} F$ is constructed as the hocolimit over $i$ of functors $T_{r}^{i} F$. In turn, each $T_{r}^{i} F(X)$ is constructed as the homotopy limit of a finite diagram of spectra obtained by applying $F$ to a finite diagram of spaces determined by $X$, and these spaces are all at least $(i-1)$-connected. The constructions are natural in both the variables $X$ and $F$, and so the next lemma follows.

Lemma 2.10. If $\Theta: C \rightarrow G$ is a stable equivalence, then

$$
P_{r} \Theta(X): P_{r} C(X) \rightarrow P_{r} G(X)
$$

is an equivalence for all $X$.

Corollary 2.11. Suppose $\Theta: C \rightarrow G$ is a stable equivalence. If, for a particular $X$, the Goodwillie tower $\left\{P_{r} C(X)\right\}$ is strongly split, then so also is the Goodwillie tower $\left\{P_{r} G(X)\right\}$. 


\section{Proof of Theorem 1.1}

The Goodwillie tower of the functor from spaces to spectra sending $X$ to $\Sigma^{\infty} \operatorname{Map}(K, X)$ consists of a diagram of functors

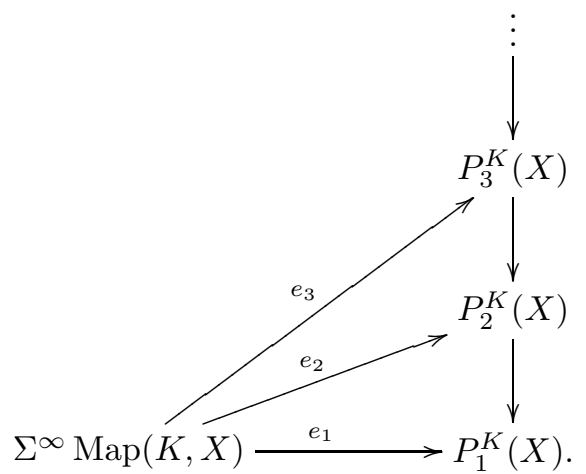

In [G2, Example 4.5], it is shown that this tower is strongly convergent if $d(K) \leq$ $c(X)$. Thus Theorem 1.1(1) will follow from Proposition 2.8 once we show that the tower is strongly split whenever $e(K) \leq s(X)$. Otherwise said, we wish to show that if $n \geq e(K)$, then the tower $\left\{P_{r}^{K}\left(\Sigma^{n} Z\right)\right\}$ is strongly split for all spaces $Z$. By Lemma 2.2, this tower agrees with the tower associated to the functor sending a space $Z$ to $\Sigma^{\infty} \operatorname{Map}\left(K, \Sigma^{n} Z\right)$.

In the terminology of the last section, the main constructions and theorems of Bö] states that if $n \geq e(K)$, then there is a filtered configuration space $C(K, Z)$ such that $\Sigma^{\infty} C(K, Z)$ is a functor with a polynomial filtration, and a natural map of spaces

$$
C(K, Z) \rightarrow \operatorname{Map}\left(K, \Sigma^{n} Z\right)
$$

such that

$$
\Sigma^{\infty} C(K, Z) \rightarrow \Sigma^{\infty} \operatorname{Map}\left(K, \Sigma^{n} Z\right)
$$

is a stable equivalence. Then Lemma 2.9 and Corollary 2.11 combine to say that the tower associated to $\Sigma^{\infty} \operatorname{Map}\left(K, \Sigma^{n} Z\right)$ is strongly split.

Statement (2) of Theorem 1.1 turns out to follow easily from statement (1). The following argument was observed by Pete Bousfield.

Suppose $g: L \rightarrow K$ is an $E_{*}$-isomorphism between finite complexes. Let $X \rightarrow$ $X_{E}$ be Bousfield localization of the space $X$ with respect to $E_{*}$ [B1].

Consider the diagram

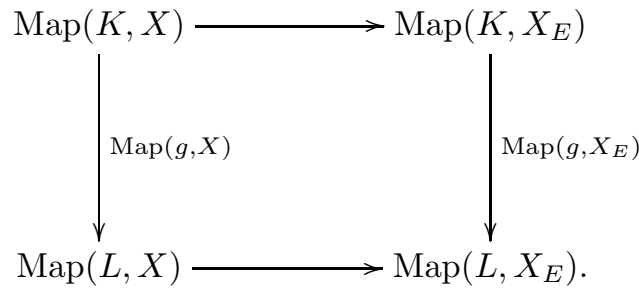

As $X \rightarrow X_{E}$ is an $E_{*}$-isomorphism, statement (1) of Theorem 1.1 applies to say that the top map is $E_{*}$-monic. The right vertical map is a homotopy equivalence as $X_{E}$ is $E_{*}$-local, and is thus an $E_{*}$-isomorphism. Thus the left vertical map is $E_{*}-$ monic. 
Remark 3.1. Though we have not needed this here, there is an explicit model for the tower $\left\{P_{r}^{K}(X)\right\}$ for $\Sigma^{\infty} \operatorname{Map}(K, X)$; see $\mathrm{Ar}, \mathrm{AK}$. From this model, it follows that a version of Corollary 2.4 holds for the $K$-variable: if $E_{*}$ is a ring theory, and $g: L \rightarrow K$ is an $E_{*}$-isomorphism, then so is $P_{r}^{f}(X): P_{r}^{K}(X) \rightarrow P_{r}^{L}(X)$. This leads to an alternative proof of Theorem $1.1(2)$, under the ring theory hypothesis.

\section{Appendix A. Computations of $e(K)$}

\section{By Greg Arone and Nicholas J. Kuhn}

Recall that $e(K)$ is the minimal $n$ such that there exists a parallelizable $n$ dimensional manifold $M$, together with a closed subcomplex $A$ such that $K \simeq M / A$.

In this Appendix we make some observations allowing for some general estimates and explicit computations of $e(K)$.

A.1. Upper bounds. If $K \simeq M / A$, with $M$ parallelizable, we will say that the pair $(M, A)$ represents $K$.

Lemma A.1. $e(K \wedge L) \leq e(K)+e(L)$.

Proof. If $(M, A)$ represents $K$ and $(N, B)$ represents $L$, then $(M \times N, A \times N \cup M \times B)$ represents $K \wedge L$.

Corollary A.2. $e\left(\Sigma^{r} K\right) \leq e(K)+r$.

Lemma A.3. Suppose that $L$ is a finite complex equivalent to a stably parallelizable $m$-manifold. If $n>m$, and $K$ is obtained from $L$ by attaching $n$-cells, then $e(K) \leq m+n$.

Proof. Suppose $g: L \rightarrow M$ is an equivalence, with $M$ a stably parallelizable $m-$ manifold. Let

$$
\coprod_{i=1}^{c} f_{i}: \coprod_{i=1}^{c} S^{n-1} \rightarrow L
$$

be the attaching maps for constructing $K$ from $L$. Then $\coprod_{i=1}^{c} S^{n-1}$ embeds in the parallelizable manifold $D^{n} \times M$ so that $\left(D^{n} \times M, \coprod_{i=1}^{c} S^{n-1}\right)$ represents $K$.

Corollary A.4. If $K$ is the mapping cone of a map $f: S^{n} \rightarrow S^{m}$, then $e(K) \leq$ $m+n+1$.

Lemma A.5. If $d(K) \geq 1$, then $e(K) \leq 2 d(K)-1$.

Proof. We can assume $K$ is a $d=d(K)$-dimensional C.W. complex. Let $L$ be its $d-1$ skeleton, and let

$$
\coprod_{i=1}^{c} f_{i}: \coprod_{i=1}^{c} S^{d-1} \rightarrow L
$$

denote the attaching maps of the $d$ - cells of $K$. The complex $L$ can be embedded in $\mathbb{R}^{2 d-1}$, and we let $U$ be a regular neighborhood. Thus $L \hookrightarrow U$ is an equivalence, and $U$ is a $2 d-1$-dimensional parallelizable manifold. The composite

$$
\coprod_{i=1}^{c} S^{d-1} \stackrel{\amalg_{i=1}^{c} f_{i}}{\longrightarrow} L \hookrightarrow U
$$

is homotopic to an embedding, and then $\left(U, \coprod_{i=1}^{c} S^{d-1}\right)$ will represent $K$. 
Slava Krushkal has told us of an unpublished result of Stallings [S] that says that any $d$-dimensional and $c$-connected finite complex is (simple) homotopy equivalent to a subcomplex of $\mathbb{R}^{2 d-c}$. This implies our final upper bound.

Lemma A.6. $e(K) \leq 2 d(K)-c(K)$.

A.2. Lower bounds. The obvious lower bound for $E(K)$ comes from dimension

$$
d(K) \leq e(K) .
$$

Stronger lower bounds arise from the contrapositive forms of the following proposition and corollary.

Proposition A.7. If $n \geq e(K)$, then $\operatorname{Map}_{\mathcal{S}}\left(K, S^{n}\right)$ is equivalent to a suspension spectrum.

Here $\operatorname{Map}_{\mathcal{S}}(K, X)$ denotes the function spectrum of stable maps between $K$ and $X$, so that $\operatorname{Map}_{\mathcal{S}}\left(K, S^{n}\right)$ is the $n$-dual of $K$.

Proof. It is easy to check (see e.g. [G1]) that the degree 1 approximation to $\Sigma^{\infty} \operatorname{Map}_{\mathcal{T}}(K, X)$ is $\operatorname{Map}_{\mathcal{S}}(K, X)$. Then, as in ${ }_{3}$, we can conclude that, if $n \geq e(K)$, then the composite

$$
\Sigma^{\infty} F_{1} C\left(K, S^{0}\right) \rightarrow \Sigma^{\infty} C\left(K, S^{0}\right) \rightarrow \Sigma^{\infty} \operatorname{Map}_{\mathcal{T}}\left(K, S^{n}\right) \rightarrow \operatorname{Map}_{\mathcal{S}}\left(K, S^{n}\right)
$$

is an equivalence, where $F_{1} C\left(K, S^{0}\right)$ is the first filtration of the configuration space $C\left(K, S^{0}\right)$.

The implications of the proposition for homology are the following.

Corollary A.8. Suppose $n \geq e(K)$.

(1) The reduced integral cohomology groups of $K$ satisfy

$$
\tilde{H}^{m}(K ; \mathbb{Z}) \text { is } \begin{cases}0 & \text { if } m>n, \\ \text { free abelian } & \text { if } m=n .\end{cases}
$$

(2) For all primes $p, \mathbb{F}_{p} \oplus \Sigma^{n} \tilde{H}_{-*}\left(K ; \mathbb{F}_{p}\right)$ admits the structure of an unstable algebra over the mod $p$ Steenrod algebra.

For certain two cell complexes, the lower bound of the proposition matches our upper bounds. Call a map $f: S^{m+k} \rightarrow S^{m}$ stably minimal if whenever $f^{\prime}: S^{m^{\prime}+k} \rightarrow$ $S^{m^{\prime}}$ is a map so that $f$ and $f^{\prime}$ represent the same element in the stable homotopy group $\pi_{k}^{S}$, one has $m \leq m^{\prime}$.

Theorem A.9. If $f: S^{n} \rightarrow S^{m}$ is stably minimal, and $K$ is the mapping cone of $f$, then $e\left(\Sigma^{r} K\right)=m+n+1+r$.

Proof. As $S$-duality induces the identity on $\pi_{*}^{S}$, one deduces that

$$
\operatorname{Map}_{\mathcal{S}}\left(\Sigma^{r} K, S^{r+m+n+1}\right) \simeq \operatorname{Map}_{\mathcal{S}}\left(K, S^{m+n+1}\right) \simeq \Sigma^{\infty} K .
$$


Since $f$ is stably minimal, $\Sigma^{-i} \Sigma^{\infty} K$ is not a suspension spectrum for any $i>0$. The proposition thus implies that $e\left(\Sigma^{r} K\right) \geq m+n+1+r$. The theorem follows, as Corollary A.4 and Corollary A.2 combine to show that $e\left(\Sigma^{r} K\right) \leq m+n+1+r$.

A.3. Examples. Theorem A.9 applies to all the classic 2-cell complexes. Explicitly, we have

(1) $e\left(M^{r+2}(d)\right)=e\left(\Sigma^{r} M^{2}(d)\right)=3+r$,

(2) $e\left(\Sigma^{r} \mathbb{C} P^{2}\right)=6+r$,

(3) $e\left(\Sigma^{r} \mathbb{H} P^{2}\right)=12+r$,

(4) $e\left(\Sigma^{r}\right.$ (Cayley plane $\left.)\right)=24+r$, and

(5) $e\left(\Sigma^{r}\left(D^{2 p+1} \cup_{\alpha} S^{3}\right)\right)=2 p+4+r$ if $p$ is an odd prime and $\alpha \in \pi_{2 p}\left(S^{3}\right)$ is an element of order $p$.

\section{ACKNOWLEDGEMENTS}

The simple argument given in 93 proving that statement (1) of Theorem 1.1 implies statement (2) is due to Pete Bousfield, and replaces a different argument by the author, which needed the side hypothesis that $E_{*}$ be a ring theory. For this, and for other encouraging 'e'-conversations, I offer Pete my thanks. Thanks are also due to my colleagues Greg Arone and Slava Krushkal for discussions about this material.

\section{REFERENCES}

[AK] S.T. Ahearn and N. J. Kuhn, Product and other fine structure in polynomial resolutions of mapping spaces, Alg. Geom. Topol. 2 (2002), 591-647. MR1917068 (2003j:55009)

[Ar] G. Arone, A generalization of Snaith-type filtration, Trans. A. M. S. 351 (1999), 11231250. MR1638238 (99i:55011)

[Bö] C.-F. Bödigheimer, Stable splitting of mapping spaces, Springer L. N. Math. 1286 (1987), 174-187. MR0922926 (89c:55011)

[B1] A. K. Bousfield, The localization of spaces with respect to homology, Topology 14 (1975), 133-150. MR0380779 (52:1676)

[B2] A. K. Bousfield, On $\lambda$-rings and the $K$-theory of infinite loop spaces, $K$-Theory 10 (1996), no. 1, 1-30. MR.1373816 (98a:55006)

[B3] A. K. Bousfield, Homotopical localizations of spaces, Amer. J. Math 119 (1997), 13211354. MR 1481817 (98m:55009)

[EKMM] A.D. Elmendorf, I. Kriz, M.A. Mandell, J.P. May, Rings, modules, and algebras in stable homotopy theory, A. M. S. Math. Surveys and Monographs 47, 1997. MR1417719 (97h:55006)

[G1] T. G. Goodwillie, Calculus I: the first derivative of pseudoisotopy, K-theory 4 (1990), 1-27. MR:1076523 (92m:57027)

[G2] T. G. Goodwillie, Calculus II: analytic functors, K-theory 5 (1992), 295-332. MR.1162445 (93i:55015)

[G3] T. G. Goodwillie, Calculus III: the Taylor series of a homotopy functor, Geom. Topol. 7 (2003), 645-711. MR2026544

[K1] N. J. Kuhn, Suspension spectra and homology equivalences, Trans. A. M. S. 283 (1984), 303-313. MR0735424 (85g:55014)

[K2] N. J. Kuhn, Localization of André-Quillen-Goodwillie towers, and the periodic homology of infinite loopspaces, Advances in Mathematics, to appear (available online).

[K3] N. J. Kuhn, Goodwillie towers and chromatic homotopy: an overview, International Conference in Algebraic Topology (Kinosaki, 2003), Geometry and Topology Monographs, to appear.

[LS] L. Langsetmo and D. Stanley, Nondurable K-theory equivalence and Bousfield localization, K-theory 24 (2001), 397-410. MR 1885129 (2002k:55008)

[Ma] J. P. May, The geometry of interated loop spaces, Springer L. N. Math. 271, 1972. $\operatorname{MR} 0420610(54: 8623 b)$ 
[McD] D. Mc Duff, Configuration spaces of positive and negative particles, Topology 14 (1975), 91-107. MR 0358766 (50:11225)

[S] J. R. Stallings, The embedding of homotopy types into manifolds, unpublished 1965 paper, available at http://math.berkeley.edu/ ${ }^{\sim}$ stall/.

Department of Mathematics, University of Virginia, Charlottesville, Virginia 22904

E-mail address: njk4x@virginia.edu 